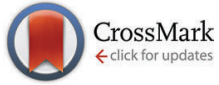

Cite this: Phys. Chem. Chem. Phys., 2016, 18, 2981

Received 12th October 2015, Accepted 19th December 2015

DOI: $10.1039 / c 5 c p 06151 b$

www.rsc.org/pccp

\section{Fluorescent DNA probes at liquid/liquid interfaces studied by surface second harmonic generation $\uparrow$}

\author{
Giuseppe Licari, ${ }^{a}$ Pierre-François Brevet ${ }^{\mathrm{b}}$ and Eric Vauthey*a
}

The properties of a series of oxazole yellow dyes, including the dicationic YOPRO-1 and its homodimeric parent YOYO-1 and two monocationic dyes (YOSAC-1 and YOSAC-3), have been investigated at the dodecane/water interface using stationary and time-resolved surface second harmonic generation (SSHG) combined with quantum chemical calculations. Whereas YOYO-1 exists predominantly as a $\mathrm{H}$-dimer in aqueous solution, the stationary SSHG spectra reveal that such dimers are not formed at the interface. No significant $\mathrm{H}$-aggregation was observed with YOPRO-1, neither in solution nor at the interface. In the case of the monocationic YOSAC dyes, a distinct SSHG band due to $\mathrm{H}$-aggregates was measured at the interface, whereas only weak aggregation was found in solution. These distinct aggregation behaviors can be explained by the different orientations of the dyes at the interface, as revealed from the analysis of polarization-resolved experiments, the doubly-charged dyes lying more flat on the interface than the singly charged ones. Although YOYO-1 and YOPRO-1 do not form H-dimer/aggregates at the interface, timeresolved SSHG measurements point to the occurrence of intra- and intermolecular interactions, respectively, which inhibit the ultrafast non-radiative decay of the excited dyes via large amplitude motion, and lead to a nanosecond excited-state lifetime. The distinct behavior evidenced here for YOYO-1 and YOSAC dyes points to their potential use as fluorescent or SHG interfacial probes.

\section{Introduction}

The synthesis of oxazole yellow (YO) cyanine dyes and their homodimeric forms (YOYO) was a major breakthrough in molecular biology, because fluorescence detection could achieve, for the first time, sensitivity to DNA similar to that of radioactive probes. ${ }^{1,2}$ Spectroscopic investigations of these cyanine dyes in solution have evidenced two mechanisms responsible for their $>100$-fold increase of fluorescence intensity upon intercalation into ds-DNA. ${ }^{3-6}$ The first arises from the loss of mobility around the methine bridge upon binding to DNA, which inhibits the non-radiative deactivation of the excited state through large amplitude motion. ${ }^{3,4,7}$ The second mechanism mostly concerns the homodimeric dyes, which tend to self-aggregate into nonfluorescent $\mathrm{H}$-dimers in aqueous solution. ${ }^{6-8}$ The excitonic interaction in the dimers results in delocalization of the electronic excitation and splitting of the energy levels into two excitonic

\footnotetext{
${ }^{a}$ Department of Physical Chemistry, University of Geneva, 30 Quai Ernest-Ansermet, CH-1211 Geneva 4, Switzerland. E-mail: eric.vauthey@unige.ch

${ }^{b}$ Institut Lumière Matière, UMR CNRS 5306, Université Claude Bernard, Lyon 1, Campus LyonTech - La Doua, 10 Rue Ada Byron, 69622 Villeurbanne cedex, France

$\dagger$ Electronic supplementary information (ESI) available: Additional SSHG spectra, polarization-resolved SSHG data, analysis of the polarization profiles, determination of the interfacial orientation, and quantum-chemical calculations. See DOI: 10.1039/c5cp06151b
}

states: a dark state at lower energy and a bright state at higher energy. ${ }^{9}$ As a consequence, $\mathrm{H}$-dimers are characterized by an absorption band that is blue-shifted relatively to that of the monomer and by an absence of fluorescence, because photoexcitation is followed by an ultrafast internal conversion from the bright to dark excitonic state. Upon binding to DNA, the dimers are disrupted, both the excitonic interaction and the non-radiative decay via large amplitude motion are suppressed, and fluorescence is operative. The removal of the excitonic interaction upon bis-intercalation into DNA was suggested to be due to not only the larger distance between the two chromophoric units but also their quasi-orthogonal mutual orientation. $^{8}$

The aforesaid ability of cyanines to self-organize is invaluable for many applications and has been extensively studied in solution, where dimers and higher-order $\mathrm{H}$ - or J-type aggregates were observed. ${ }^{10-18}$ Moreover, the aggregation properties of several cyanine derivatives were also studied at liquid/water or air/water interfaces using surface second harmonic generation (SSHG) ${ }^{19-26}$ Steady-state, time-resolved (TR) SSHG as well as SHG microscopy were applied to obtain information about their adsorption, orientation and dynamic properties. These studies revealed that cyanines, like other surface-active organic molecules ${ }^{27-32}$ form aggregates at the interface already at concentrations at which no aggregation takes place in the bulk phase. 
<smiles>C[n+]1c(C=C2C=CN(CCC[N+](C)(C)CCCN3C=CC(=Cc4oc5ccccc5[n+]4C)c4ccccc43)c3ccccc32)oc2ccccc21</smiles><smiles></smiles>

YOPRO-1
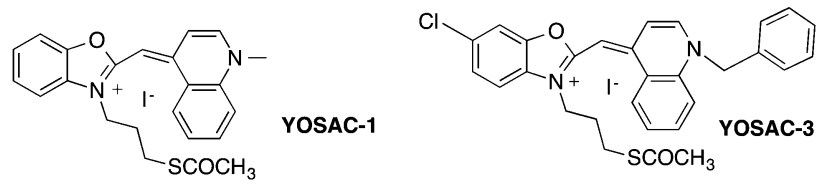

Chart 1 Structure of the fluorescent DNA probes.

To the best of our knowledge, YO and YOYO DNA probes have never been investigated so far at liquid/water interfaces. Additionally to exhibit fluorescence enhancement upon intercalation into DNA, a good fluorescent probe should be soluble in water and have a high affinity for the low-polarity environment created by the DNA base-pair stack. Therefore, fluorescence DNA intercalators can be expected to have a high affinity for liquid/water interfaces. To test this idea and evaluate whether such dyes could be good interfacial probes, we have carried out an investigation of four cyanine dyes belonging to the YO family (Chart 1) at the dodecane/water interface using both stationary and time-resolved SSHG measurements combined with quantum chemical calculations. These dyes differ in their electric charge, the number of chromophoric units and the hydrophobic substituents. YOPRO-1 bears two positive charges, whereas its homodimeric relative YOYO-1 has four positive charges. YOSAC-1 and YOSAC- 3 are only singly charged and possess a hydrophobic chain that should confer them higher surface-activity. Here, we show that these dyes generate intense resonantly enhanced SSHG signal at the dodecane/water interface that could make them attractive two-photon probes for biological imaging in the near infrared region. ${ }^{33-35}$ Furthermore, their aggregation behaviour is strongly dye-dependent. With YOYO-1, the H-dimer form that predominates in aqueous solution is no longer present at the interfaces. On the other hand, the YOSAC dyes exhibit a much higher propensity to aggregate at the interface than in solution. These results will be rationalized in terms of different orientations of the dyes when adsorbed at the interface.

\section{Experimental}

\section{Samples}

The dyes were obtained from Prof. T. Deligeorgiev (Faculty of Chemistry, University of Sofia) and were used as received. Their synthesis has been described in ref. 36 and 37. Stock solutions of the dyes were prepared in DMSO (Acros Organics, spectroscopic grade) at $2 \mathrm{mM}$ concentration and the samples were freshly prepared from the stock solutions before each measurement. Dodecane (DOD, 99+\%) was purchased from Alfa Aesar, phosphate-buffered saline (PBS, composition: $2.7 \mathrm{mM} \mathrm{KCl}$, $1.5 \mathrm{mM} \mathrm{KH} \mathrm{PO}_{4}, 137.9 \mathrm{mM} \mathrm{NaCl}$, and $8.1 \mathrm{mM} \mathrm{Na}_{2} \mathrm{HPO}_{4} \cdot 7 \mathrm{H}_{2} \mathrm{O}$ ) from Invitrogen and ethylenediaminetetraacetic acid (EDTA) disodium salt dihydrate from Applichem. The PBS solutions also contained $1 \mathrm{mM}$ EDTA. Double-stranded salmon sperm DNA was purchased from Sigma.

Absorption spectra were recorded on a Cary 50 spectrophotometer (Varian) using $1 \mathrm{~cm}$ quartz cells. The samples for SSHG measurements were prepared by pouring $10 \mathrm{~mL}$ of aqueous dye solution into a $4 \times 4 \times 4 \mathrm{~cm}^{2}$ quartz cell and then slowly adding $12 \mathrm{~mL}$ of dodecane. The experiments were performed at $20 \pm 1{ }^{\circ} \mathrm{C}$. For concentration-dependent SSHG measurements, the concentration was changed in situ by adding variable amounts of dye into the sample with a syringe. Such an addition leads to a rise of the interface plane by at most $60 \mu \mathrm{m}$. With the $500 \mathrm{~mm}$ lens used to focus the probe beam, the Rayleigh length is around $20 \mathrm{~mm}$. Therefore, such a small change in the position of the interface has a negligible effect on the SSHG signal.

\section{SSHG measurements}

The SSHG setup has been already described in detail previously. ${ }^{38,39}$ Shortly, the near-infrared probe pulses $(\sim 100 \mathrm{fs}$, $\sim 1 \mu \mathrm{J}$ at the interface) were generated using a collinear optical parametric amplifier (TOPAS-C, Light Conversion) and their polarization was controlled by a half-wave plate. They were focused onto the interface at an angle of incidence of about $70^{\circ}$. The pump pulses ( $\sim 80 \mathrm{fs}, \sim 1 \mu \mathrm{J}$ at the interface) used in TR-SSHG were produced by a non-collinear optical parametric amplifier (TOPAS White, Light Conversion) and were circularly polarized to avoid photoselection. Both TOPASs were fed by the output of a $1 \mathrm{kHz}$ Ti:Sapphire amplified system (Solstice, Spectra-Physics) centred at $800 \mathrm{~nm}$. The SSHG signal was focused onto the entrance slit of a Czerny-Turner spectrograph (Shamrock 163, Andor) and detected by a multipixel cooled CCD camera (Newton 920, Andor). The SSHG intensity was checked to scale quadratically with the probe intensity. The non-resonant signal coming from the bare interface was found to be negligibly small with respect to the resonant signal coming from the dye and was not subtracted. The raw TR-SSHG profiles were transformed into $S(t)$ profiles by first taking the square root of the measured signal to obtain a quantity that scales linearly with the concentration of the probed molecule as well as with the modulus of the second-order nonlinear susceptibility, $\left|\chi^{(2)}\right|$. This procedure could be done here because the SSHG signal is purely resonant. The photoinduced change in the resulting intensity was then normalized to -1 , since in the present measurements, photoexcitation always resulted in a decrease of the signal. The usual polarization nomenclature will be used: $\mathrm{p}$ for parallel polarization with respect to the plane of incidence and $\mathrm{s}$ for perpendicular polarization.

The SSHG spectra were recorded by scanning the TOPAS-C with 2 or $2.5 \mathrm{~nm}$ steps. The SSGH spectra of the dyes were corrected for the wavelength dependence of the setup using as reference the SSHG signal measured with a silver mirror immersed in dodecane (Thorlabs, see reflectance in Fig. S11 in the ESI†). 
If not specified, the SSHG spectra were recorded at $\mathrm{m} / \mathrm{s}$ polarization geometry, where $\mathrm{m}$ stands for mixed polarization $\left(45^{\circ}\right.$ with respect to the plane of incidence) and designates the polarization of the probe field, whereas $s$ is the measured polarization component of the SSHG signal. This geometry was chosen arbitrarily among the three most used polarization geometries (m/s, p/p and s/p). Furthermore, the raw SSHG spectra were transformed into $S(\lambda)$ spectra by taking the square root of intensity and by subsequent normalization, so that $S(\lambda)$ is proportional to $\left|\chi^{(2)}\right|$.

\section{Quantum chemical calculations}

All the calculations were done at the density functional theory (DFT) level using the Gaussian 09 (Rev. C) package. ${ }^{40}$ For geometry optimizations, the Becke's three-parameter exchange functional $^{41}$ and the Lee-Yang-Parr (LYP) correlation functional in the form of the B3LYP functional, ${ }^{42}$ as well as tight convergence criteria were employed. For the prediction of electronic response properties, such as vertical excitations and hyperpolarizabilities, the Coulomb-attenuating hybrid exchangecorrelation functional (CAM-B3LYP) with improved long-range properties was used with the following functional parameters: $\mu=0.33, \alpha=0.15$ and $\beta=0.37 .{ }^{43}$ This method has already been successfully used to compute the electronic (hyper)polarizability of organic compounds. ${ }^{44-47}$ Moreover, contrary to the B3LYP functional, it does not predict low-lying charge-transfer states for the molecules investigated here. As an extended basis set is required for computing hyperpolarizability, ${ }^{48-50}$ the Pople 6-311++G(d,p) basis set with diffuse and polarization functions was used throughout. Water solvation was treated implicitly through the SMD model proposed by Truhlar and coworkers based on the integral-equation-formalism polarized continuum model (IEF-PCM) implemented in Gaussian 09. ${ }^{51}$ The structure of the YO monomer was re-optimized at CAMB3LYP/6-311++G(d,p) in order to obtain the ground-state transition dipole moment at the same level of theory as the hyperpolarizability.

The electronic transition energies were obtained from timedependent DFT (TD-DFT), ${ }^{52-54}$ including solvent effects with the PCM model. Frequency-dependent hyperpolarizabilities, $\beta(-2 \omega ; \omega, \omega)$, were computed in the EFISH orientation through the Coupled-Perturbed Hartree-Fock procedure. ${ }^{55-57}$ A comprehensive discussion on the second-order optical response can be found in ref. 50. For each hyperpolarizability tensor element, a total number of 80 frequencies were calculated in a range centred at the most intense vertical transition predicted by the TD-DFT calculations. The near-IR spectral ranges were adapted according to the system: 0.0520-0.0582 a.u. (1.41-1.58 eV) for YO, 0.0530-0.0595 a.u. (1.44-1.62 eV) for the H-dimer and 0.0516-0.0580 a.u. (1.40-1.58 eV) for the J-dimer. The real part of the hyperpolarizability obtained from the calculations was converted into the imaginary part using the KramersKronig relationship. Only the electronic contribution to the first hyperpolarizability was considered and the pure vibrational and zero-point vibrational average contributions were neglected.

\section{Results and discussion}

\section{SSHG spectra}

The electronic absorption spectra of diluted aqueous solutions of YO cyanine dyes are characterized by a low-energy band arising from the $S_{1} \leftarrow S_{0}$ transition with a maximum between 470 and $490 \mathrm{~nm}$ and with a shoulder at shorter wavelength due to a vibronic transition. ${ }^{3}$ Very similar spectra are observed with the homodimer YOYO-1 in organic solvents (Fig. 1 and 2). ${ }^{6-8}$ In aqueous solution, however, the absorption band culminates at $458 \mathrm{~nm}$ with a secondary maximum at $485 \mathrm{~nm}$. The $458 \mathrm{~nm}$ band has been shown to originate from intramolecular YOYO-1 $\mathrm{H}$-dimers absorbing coincidentally at a wavelength similar to that of the vibronic transition of the non-aggregated form. ${ }^{6-8}$ Addition of ds-DNA results in the suppression of the $\mathrm{H}$-dimer band and in a spectrum close to that measured in organic solvents. This effect arises from the bis-intercalation of YOYO-1 into DNA and the ensuing elimination of the excitonic interaction between the two chromophoric units. ${ }^{8}$ Aggregation can also be observed, although to a smaller extent, with the YOSAC dyes as a weak growth of the relative absorbance on the blue side of the absorption band upon increasing concentration (Fig. S4, ESI $\dagger$ ).

Fig. 2 shows a comparison of the electronic absorption and SSHG spectra, $S(\lambda)$, of the dyes in aqueous solution and the dodecane/water interface, respectively. The measurements with YOPRO-1 were performed in PBS to obtain a higher SSHG signal (see below). In principle, the SSHG spectrum should be compared with the product of the one- and two-photon absorption spectra. ${ }^{58}$ However, one- and two-photon absorption spectra are very similar for molecules far from being centrosymmetric, ${ }^{59-61}$ which are thus polar and characterized by a significant hyperpolarizability, $\beta$. Since the cyanines studied here are polar and have a substantial $\beta$ (see below), the SSHG spectra can be safely compared with the linear absorption spectra. ${ }^{62}$

Fig. 2 reveals that the SSHG spectrum of YOYO-1 lacks the $458 \mathrm{~nm}$ band of the $\mathrm{H}$-dimer and resembles the electronic absorption spectrum measured in organic solvents or in the presence of DNA, where YOYO-1 is in the non-aggregated form.

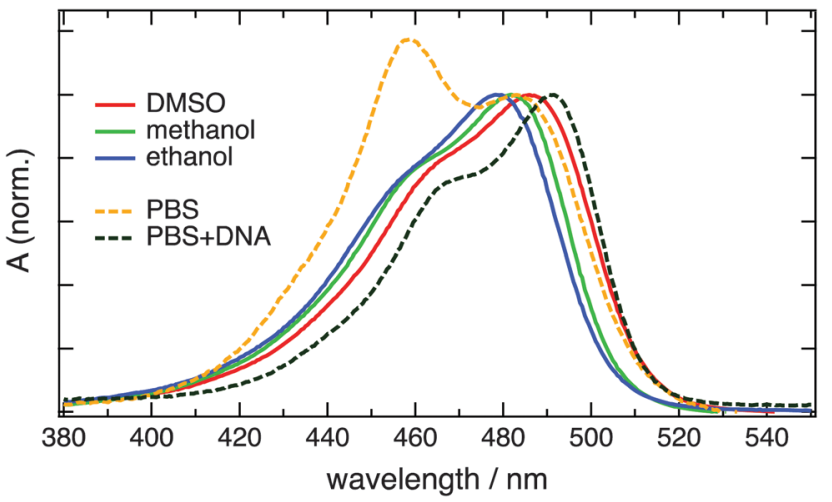

Fig. 1 Electronic absorption spectra measured with $2 \mu \mathrm{M}$ YOYO-1 in various solvents. The concentration of DNA corresponds to a base-pair/ dye ratio $>5$ 


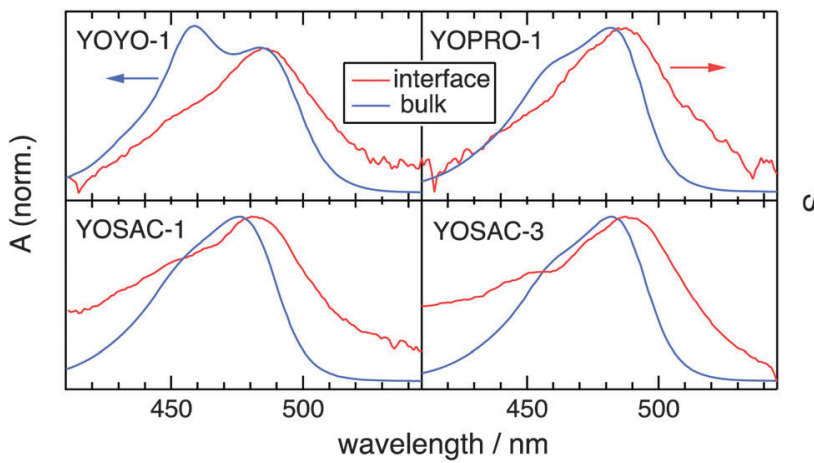

Fig. 2 Comparison of the electronic absorption and SSHG spectra, $S(\lambda)$, of the YO dyes in aqueous solution and at the dodecane/water interface, respectively. The aqueous phase is water for all compounds except for YOPRO-1 for which PBS was used. The concentration was $10 \mu \mathrm{M}$ for YOPRO-1 and $5 \mu \mathrm{M}$ for the other dyes.

This indicates that YOYO-1 does not form H-dimers when adsorbed at the interface. An alternate explanation, which can be dismissed on the basis of quantum-chemical calculations described below, would be that the interfacial $\mathrm{H}$-dimers make a negligibly small contribution to the SSHG signal. The SSHG spectrum recorded with YOPRO-1 is very similar to that of YOYO-1. The small bathochromic shift of the band maximum relative to the absorption band could be due to a solvatochromic effect, in agreement with the solvent dependence of the absorption spectrum shown in Fig. 1. This indicates that, like in bulk aqueous solution, YOPRO-1 does not form $\mathrm{H}$-aggregates at the interface.

A different behaviour is observed with the YOSAC dyes (Fig. 2): in addition to a red-shifted maximum and a broadening on the long-wavelength side that is larger than that observed for the two other cyanines, the SSHG band is markedly more intense at wavelengths corresponding to the absorption of the H-dimer, pointing to the formation of $\mathrm{H}$ aggregates at the interface.

To support the assignment of this spectral broadening to aggregation, the SSHG spectra of all four dyes were recorded at different bulk concentrations and are depicted in Fig. 3, and Fig. S3 and S4 (ESI $\dagger$ ). The shape of the SSHG spectra recorded with YOYO-1 is independent of concentration (Fig. 3A), indicating that this dye forms neither intramolecular $\mathrm{H}$-dimers nor intermolecular $\mathrm{H}$-aggregates at the interface, at least at those concentrations. In the case of YOPRO-1, the relative intensity of the blue side of the band does not depend on concentration either, confirming the absence of $\mathrm{H}$-aggregation at the interface. A small increase of the relative intensity on the red side of the spectrum appears (>500 nm) when going from 10 to $15 \mu \mathrm{M}$ YOPRO-1. This difference is however small and not far from the noise level of the signal and vanishes if the aqueous solution is stirred during measurements. Such stirring decreases the signal to noise ratio and was thus not systematically performed as it requires longer acquisition time.

Fig. 3B reveals that the SSHG spectrum recorded with YOSAC-1 changes dramatically with increasing concentration and, at $20 \mu \mathrm{M}$, exhibits a distinct secondary maximum at around $453 \mathrm{~nm}$ that

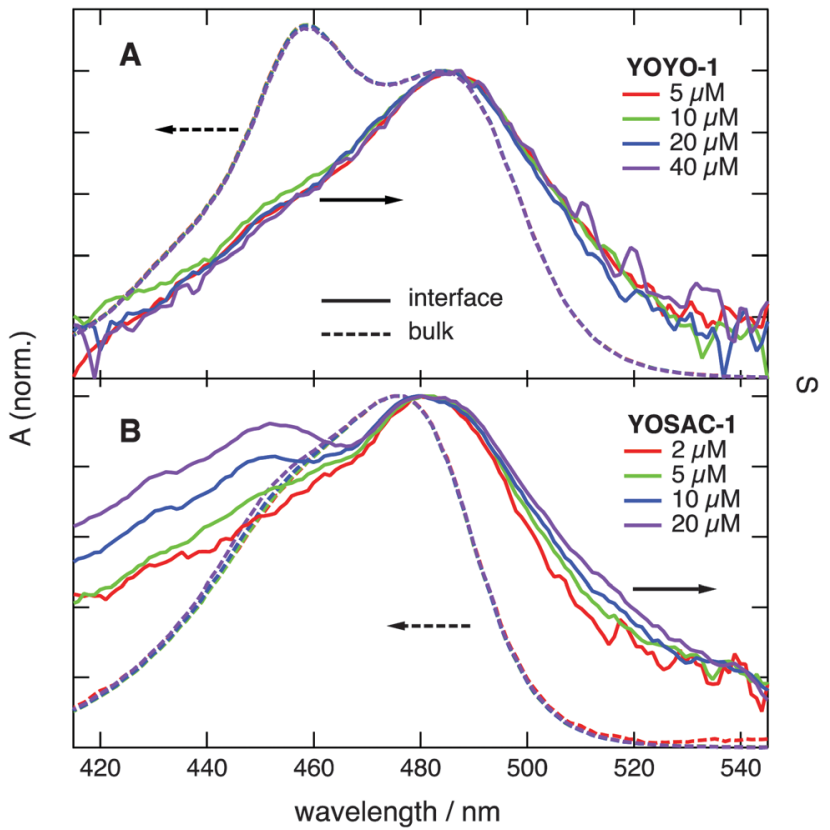

Fig. 3 Concentration dependence of the SSHG spectra, $S(\lambda)$, measured with (A) YOYO-1 and (B) YOSAC-1 at the dodecane/water interface. The electronic absorption spectra in water at the same concentrations are shown for comparison.

coincides with the H-dimer absorption band measured with YOYO-1 in aqueous solution. Additionally, the relative SSHG intensity on the red side of the band exhibits a weaker but distinct growth with increasing concentration. As this increase seems to follow the same concentration dependence on both sides of the band, it should be associated with a single type of aggregate rather than to the coexistence of both $\mathrm{H}$ - and J-type aggregates at the interface. $\mathrm{H}$ - and J-aggregates are two extreme cases where the arrangement of the chromophores is such that it leads to either cancellation or addition of the dipolar strength, i.e. to totally dark and bright excitonic states. ${ }^{9}$ The weaker increase of the red side of the band relative to the blue side could be due to aggregates with a mutual orientation of the chromophores departing from that in an ideal $\mathrm{H}$-aggregate, and which, therefore, does not lead to totally dark lower excitonic states.

Increasing YOSAC-3 concentration affects only the blue-side of the SSHG band (Fig. S4A, ESI $\dagger$ ), but to a much smaller extent compared to YOSAC-1. Indeed, whereas a clear secondary maximum is observed with $20 \mu \mathrm{M}$ YOSAC-1, only a shoulder can be seen with the same concentration of YOSAC-3.

The higher propensity of the YOSAC dyes compared to YOYO-1 and YOPRO-1 to form H-aggregates at the interface can be explained by their lower electric charge, which first decreases their hydrophilic character, favouring their adsorption at the interface, and second, decreases Coulombic repulsion, abetting aggregation. The higher relative contribution of the $\mathrm{H}$-aggregate to the SSHG signal observed with YOSAC-1 relative to YOSAC-3 could reflect a higher propensity of the former to aggregate. This could arise from the bulky phenyl-methylene 
substituent on YOSAC-3, which albeit it enhances the hydrophobic character of this dye and most likely its affinity for the interface, most probably disfavours aggregation through steric hindrance. However a large hyperpolarizability of the YOSAC-1 aggregate or a different orientation at the interface cannot be excluded.

The SSHG measurements with YOPRO-1 were performed in PBS, because the signal in pure water was too weak to record reliable spectra, in agreement with the above discussion about the influence of electric charge on the interfacial activity. The SSHG intensity was found to substantially increase for all four dyes when going from pure water to PBS. This effect has already been reported, ${ }^{30,38,63}$ and is due to the higher affinity of some ions, like $\mathrm{Cl}^{-}$, for the interface and thus the Coulombic attraction of the cationic cyanine dyes toward the interfacial region.

Using the buffer instead of pure water has no significant effect on the electronic absorption and SSHG spectra of YOYO-1 and YOSAC-1 (Fig. S1 and S2, ESI $\dagger$ ). On the other hand, the electronic absorption spectrum of YOSAC-3 in PBS exhibits an additional band at around $535 \mathrm{~nm}$ whose relative intensity increases with dye concentration, and which can be attributed to a J-aggregate (Fig. S4B, ESI $\dagger$ ). This effect, not observed with other dyes, confirms the more hydrophobic nature of YOSAC-3. However, this red-shifted band does not appear in the SSHG spectrum at the dodecane/PBS interface (Fig. S4B, ESI $\dagger$ ). The shoulder at around $450 \mathrm{~nm}$, due to the $\mathrm{H}$-aggregates, can still be observed but is weaker and increases less with concentration than at the dodecane/water interface. These results indicate that the buffer does not affect very significantly the aggregation behaviour of the YO dyes at the interface.

Whereas the enhanced aggregation of the YOSAC dyes at the interface can be explained in terms of higher interfacial population due to their superior amphiphilic character, the absence of YOYO-1 H-dimers cannot be accounted for by concentration effects, because this $\mathrm{H}$-dimer is intramolecular and its formation does not depend on concentration. Therefore, the lack of a $\mathrm{H}$-dimer band should be rather due to the mutual orientation of the two YO units when adsorbed at the interface that does not lead to significant excitonic coupling.

\section{Polarization-resolved SSHG and orientation at the interface}

To obtain a deeper insight into the interfacial orientation of the dyes, polarization-resolved SSHG measurements were carried out with YOYO-1 and YOSAC-1. In a first step, SSHG spectra were recorded at different polarizations to evidence possible differences between the nonlinear susceptibility tensors associated with the aggregated and non-aggregated forms of the dye, $\overleftrightarrow{\chi}_{a}^{(2)}$ and $\overleftrightarrow{\chi}_{n}^{(2)}$, respectively.

The shape of the SSHG spectrum measured with YOYO-1 does not exhibit a significant dependence on the polarization of the probe beam ( $\mathrm{s}, \mathrm{p}$, or $\mathrm{m}$ ) and the measured polarization component of the signal ( $\mathrm{s}$ or p) (Fig. S5, ESI $\dagger$ ). This indicates that, unless the relative magnitudes of the tensor elements of $\overleftrightarrow{\chi}_{a}^{(2)}$ and $\overleftrightarrow{\chi}_{n}^{(2)}$ are identical, which is improbable as shown below, only one species, namely the non-aggregated form, contributes to

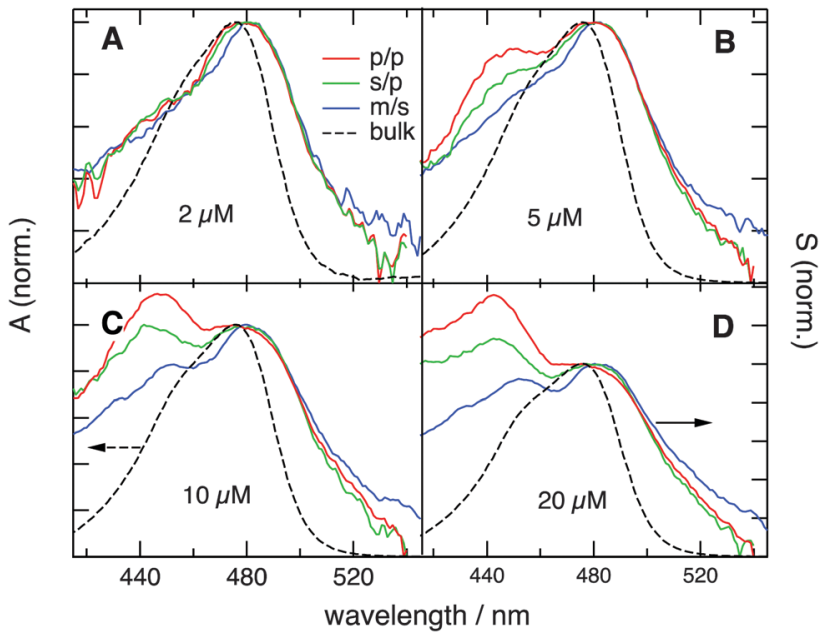

Fig. 4 SSHG spectra measured with YOSAC-1 at different concentrations and different polarization geometries. The electronic absorption spectra are shown for comparison.

the SSHG signal. Much stronger polarization dependence, which increases substantially with concentration, is observed with YOSAC-1 as shown in Fig. 4. At $2 \mu \mathrm{M}$, the H-aggregate band is absent and the shape of the SSHG spectrum is the same for all three polarization geometries, indicating that the signal originates from one species only. At higher concentrations, a strong polarization dependence of the spectral shape can be observed. These changes are the largest below $480 \mathrm{~nm}$, but can also be detected on the long-wavelength side of the band. As already suggested above, the SSHG intensity on the red side of the band could be due to a resonance with the transition to the lowest excitonic state of the aggregate, which is not totally dark due to small departure from the ideal $\mathrm{H}$-aggregate geometry. In the $\mathrm{p} / \mathrm{p}$ geometry and above $10 \mu \mathrm{M}$, the $\mathrm{H}$-aggregate band at around $450 \mathrm{~nm}$ is substantially more intense than the band due to the non-aggregated dyes.

The three polarization geometries probe different elements of the susceptibility tensor. In the case of an isotropic interface with $C_{\infty \mathrm{v}}$ symmetry, the nonlinear susceptibility tensor contains only three independent sets of nonzero elements: $\chi_{Z Z Z}^{(2)}$, $\chi_{Z X X}^{(2)}=\chi_{Z Y Y}^{(2)}$ and $\chi_{X X Z}^{(2)}=\chi_{X Z X}^{(2)}=\chi_{Y Z Y}^{(2)}=\chi_{Y Y Z}^{(2)}$ where the subscripts are the Cartesian coordinates in the laboratory frame, $X$ and $Y$ being in the interfacial plane and $Y$ and $Z$ in the plane of incidence of the probe beam. With the geometry used here, the dependence of the SSHG intensity on the polarization angle of the probe beam, $\gamma\left(\gamma=0^{\circ}, 45^{\circ}\right.$ and $90^{\circ}$ for $\mathrm{p}, \mathrm{m}$ and $\mathrm{s}$ polarization $)$, and of the signal component, $\Gamma$ ( $\Gamma=0$ and $90^{\circ}$ for $\mathrm{p}$ and s components) is given by: ${ }^{64,65}$

$$
\begin{aligned}
I_{\mathrm{SSHG}}(\gamma, \Gamma)= & C \mid a_{1} \chi_{X X Z}^{(2)} \sin 2 \gamma \sin \Gamma+\left(a_{2} \chi_{X X Z}^{(2)}+a_{3} \chi_{Z X X}^{(2)}\right. \\
& \left.+a_{4} \chi_{Z Z Z}^{(2)}\right) \cos ^{2} \gamma \cos \Gamma+\left.a_{5} \chi_{Z X X}^{(2)} \sin ^{2} \gamma \cos \Gamma\right|^{2} I_{\mathrm{pr}}{ }^{2}
\end{aligned}
$$

where $C$ is a constant that depends on the solvents and the probe wavelength, $a_{1}-a_{5}$ are optical coefficients that depend on 
the relative dielectric constant of the solvents and the experimental angle of incidence (see ESI $\dagger$ ), and $I_{\mathrm{pr}}$ is the intensity of the probe field. Under the experimental conditions used here, both $a_{2}$ and $a_{3}$ are close to zero (Table S1, ESI $\dagger$ ), and consequently, the three different polarization geometries probe the three independent susceptibility elements selectively:

$$
\begin{gathered}
I_{\mathrm{SSHG}}^{\mathrm{m} / \mathrm{s}}=I_{\mathrm{SSHG}}\left(45^{\circ}, 90^{\circ}\right) \propto\left|a_{1} \chi_{X X Z}^{(2)}\right|^{2} \\
I_{\mathrm{SSHG}}^{\mathrm{p} / \mathrm{p}}=I_{\mathrm{SSHG}}\left(0^{\circ}, 0^{\circ}\right) \propto\left|a_{4} \chi_{Z Z Z}^{(2)}\right|^{2} \\
I_{\mathrm{SSHG}}^{\mathrm{S} / \mathrm{p}}=I_{\mathrm{SSHG}}\left(90^{\circ}, 0^{\circ}\right) \propto\left|a_{5} \chi_{Z X X}^{(2)}\right|^{2}
\end{gathered}
$$

Fig. 4 shows that the $\mathrm{H}$-aggregate band is the most visible with the $\mathrm{p} / \mathrm{p}$ geometry. This indicates that the nonlinear susceptibility tensors of the aggregated and non-aggregated forms of YOSAC-1, $\overleftrightarrow{\chi}_{a}^{(2)}$ and $\overleftrightarrow{\chi}_{n}^{(2)}$, are not identical and differ by the relative size of their elements, $\chi_{Z Z Z}^{(2)}$ being comparatively larger for the aggregate. The nonlinear susceptibility is a macroscopic quantity that depends on the population, $N_{i}$, and hyperpolarizability, $\beta_{i}$, of different species $i$ adsorbed at the interface and contributing to the signal: ${ }^{64}$

$$
\overleftrightarrow{\chi}^{(2)}=\sum_{i} \overleftrightarrow{\chi}_{i}^{(2)} \propto \sum_{i} N_{i}\langle\overleftrightarrow{\beta}\rangle
$$

where the brackets indicate an average of the molecular orientations at the interface. The average orientation can be estimated from the relative magnitudes of the tensor elements of $\overleftrightarrow{\chi}_{i}^{(2)}$ and $\overleftrightarrow{\beta}_{i}$. The $\overleftrightarrow{\chi}_{i}^{(2)}$ elements can be determined from polarizationresolved SSHG measurements. ${ }^{28,64-69}$ In a standard experiment, one records the intensity of several polarization components of the SSHG signal, e.g. $\Gamma=0^{\circ}(\mathrm{p})$ and $90^{\circ}(\mathrm{s})$, as a function of the polarization of the probe beam $\gamma$, (Fig. 5), and analyses the

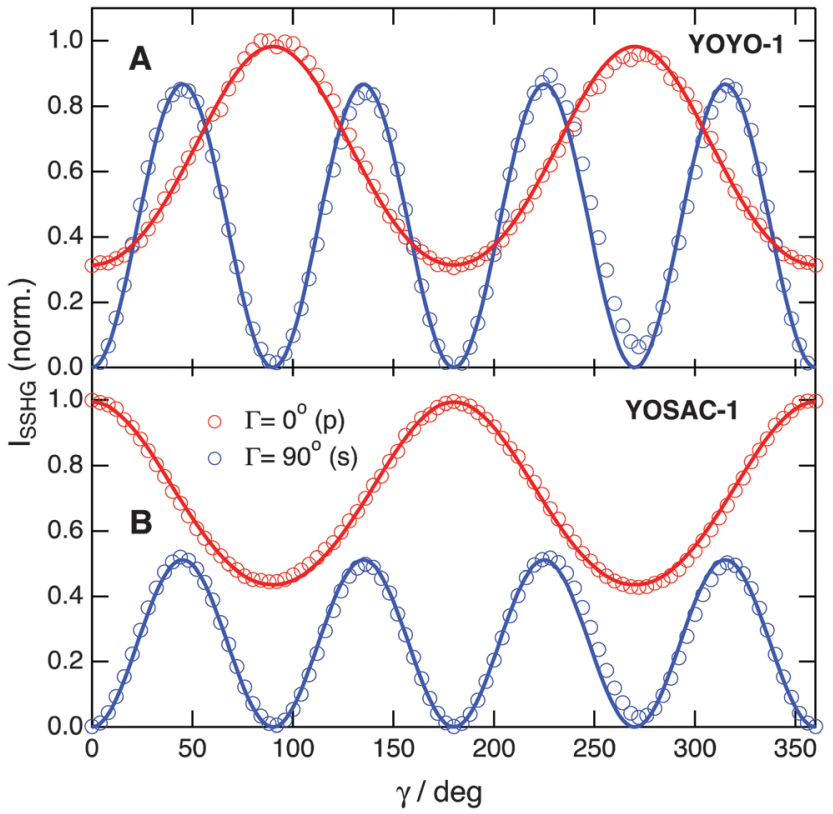

Fig. 5 Polarization profiles measured with $10 \mu \mathrm{M}$ YOYO-1 (A) and YOSAC-1 (B) and recorded at $\Gamma=0^{\circ}\left(\mathrm{p}\right.$, red) and $\Gamma=90^{\circ}$ (s, blue) upon probing at $1000 \mathrm{~nm}$ resulting intensity profiles using eqn (1). Analysis of an additional polarization profile measured at e.g. $\Gamma=45^{\circ}$ gives access to the relative sign of the susceptibility tensor elements.

The relative magnitude of the $\stackrel{\leftrightarrow}{\beta}$ elements can be determined using quantum-chemical calculations or can be estimated on the basis of the structure and symmetry of the molecule. Here, we assume that $\overleftrightarrow{\beta}$ is dominated by a single element, namely $\beta_{z z z}$, where the subscript corresponds to the Cartesian coordinates in the molecular frame, and where the $z$ axis coincides with the $\mathrm{S}_{1} \leftarrow \mathrm{S}_{0}$ transition dipole moment, which itself is aligned along the long molecular axis (see Fig. 7). The validity of this assumption is supported by the quantum-chemical calculations described in the next section and by the ratio of the hyperpolarizability tensor elements extracted from the polarization profiles (Table S2, ESI $\dagger$ ). Polarization profiles similar to those shown in Fig. 5 have been recorded with YOYO-1 and YOSAC-1 at four different concentrations and at 880 and $1000 \mathrm{~nm}$, where the aggregated and nonaggregated forms of the dyes should contribute most to the signal, respectively, (Fig. S6 and S7, ESI $\dagger$ ).

These profiles were analysed as described in detail in the $\mathrm{ESI} \dagger$ to determine the orientation parameter, $D=\left\langle\cos ^{3} \theta\right\rangle /\langle\cos \theta\rangle$, where $\theta$ is the tilt angle, i.e. the angle between the $z$ molecular axis and the normal of the interface. The tilt angle $\theta$ was then calculated assuming a Dirac delta-function distribution of orientations at the interface. Fig. 6 shows the dependence of $\theta$ on the concentration of YOYO-1 and YOSAC-1 determined from the SSHG measurements at 880 and $1000 \mathrm{~nm}$. The absolute values of $\theta$ should not be taken too literally and should be considered as higher-limit values as they were determined assuming a Dirac delta-function distribution of orientations. The same orientation parameter $D$ can be obtained assuming a Gaussian distribution of the tilt angle centred around a smaller $\theta$. Despite this, the following information can be extracted from this figure:

(1) At all concentrations and probe wavelengths, the tilt angle of YOYO-1 is substantially larger than that of YOSAC-1, indicating that the two chromophoric units of YOYO-1 lie flatter on the dodecane/water interface than YOSAC-1. This can be

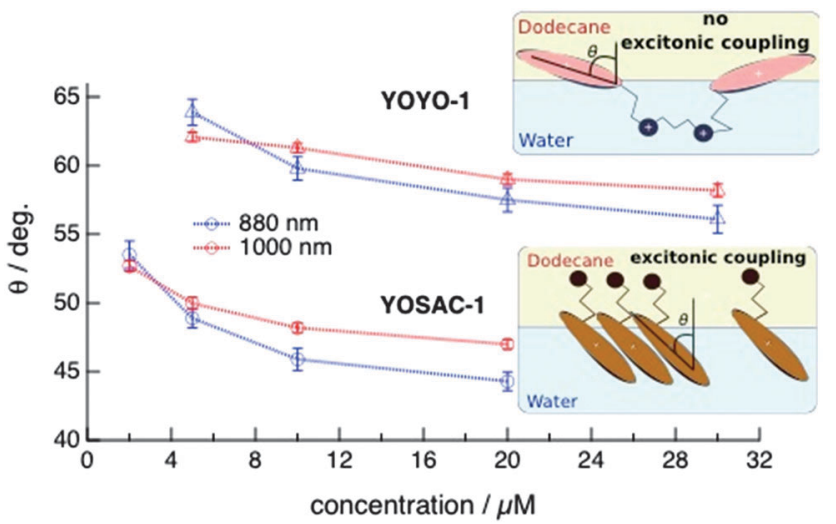

Fig. 6 Tilt angle, $\theta$, determined from polarization-resolved SSHG measurements at 880 and $1000 \mathrm{~nm}$ as a function of the concentration of YOYO-1 and YOSAC-1 in the aqueous phase. The dotted lines are guide for the eye. The insets show schematic representations of the interfacial orientation. 
understood by considering the distribution of the electric charges on YOPRO-1, which corresponds to one half of YOYO-1, with one positive charge located on the ammonium group and the other delocalized over the whole aromatic system. Consequently, the ammonium end should be attracted toward the aqueous phase, whereas the aromatic system with its delocalized charge is neither highly hydrophilic nor lipophilic. A flat orientation at the interface of the chromophoric unit can accommodate this charge distribution.

By contrast, YOSAC-1 has a larger amphiphilic character as it contains a lipophilic side-chain that should be attracted toward the apolar phase. The presence of this chain most probably favours a more perpendicular orientation with respect to the interface.

(2) For both dyes, increasing concentration leads to a decrease of $\theta$, i.e. to a more perpendicular orientation. This could be explained by considering that a smaller tilt angle allows a denser packing of adsorbates at the interface. This effect is less pronounced with YOYO-1, most probably because of the charge distribution discussed above that prevents the perpendicular orientation. The latter would require a hydrophilic fragment of the molecule to reside in the apolar phase.

(3) In the case of YOYO-1, the wavelength dependence of $\theta$ and of its variation with concentration are small and close to the limit of error. It could arise from the neglect of dispersion in the calculation of the optical coefficients $a_{i}$ (see ESI $\dagger$ ). In any case, this confirms that the SSHG signal is mainly due to a single form of the molecule and that H-dimers do not play a significant role at the interface. The more distinct wavelength dependence that can be observed with YOSAC-1 above $\sim 5 \mu \mathrm{M}$ is in full agreement with the SSHG spectra, which exhibits the $\mathrm{H}$-aggregate band at around $445 \mathrm{~nm}$ at $5 \mu \mathrm{M}$ and higher concentrations. The tilt angle obtained from the $880 \mathrm{~nm}$ data is significantly smaller than that deduced from the polarization profiles at $1000 \mathrm{~nm}$, pointing to a more perpendicular orientation of the aggregates relatively to the monomeric YOSAC-1 molecules. This is in excellent agreement with $\mathrm{H}$-aggregation, which requires a face-to-face arrangement of the dyes that cannot be achieved with a large tilt angle, i.e. with the molecules lying on the interface.

\section{Calculations of the frequency-dependent hyperpolarizability}

In order to have a better insight into the SSHG response of the aggregates, quantum-chemical calculations of the hyperpolar-

izability tensor, $\stackrel{\leftrightarrow}{\beta}$, of the monomeric and dimeric forms of the YO dyes were performed at the DFT level, using a YO analogue with methyl substituents on the two nitrogen atoms (Fig. 7). A solvation model for water was included in the calculation to better describe the experimental conditions (see Experimental section). The molecular coordinates were fixed in all calculations with the $z$-axis aligned along the $S_{1} \leftarrow S_{0}$ transition dipole moment of YO. Geometries of two dimers among the optimized ones were identified as $\mathrm{H}$ - and J-dimers (Fig. 7). The rotational offset (i.e. the angle between the long axes of the two YO units) amounts to $20^{\circ}$ and $39^{\circ}$ for the $\mathrm{H}$ - and J-dimers, whereas the

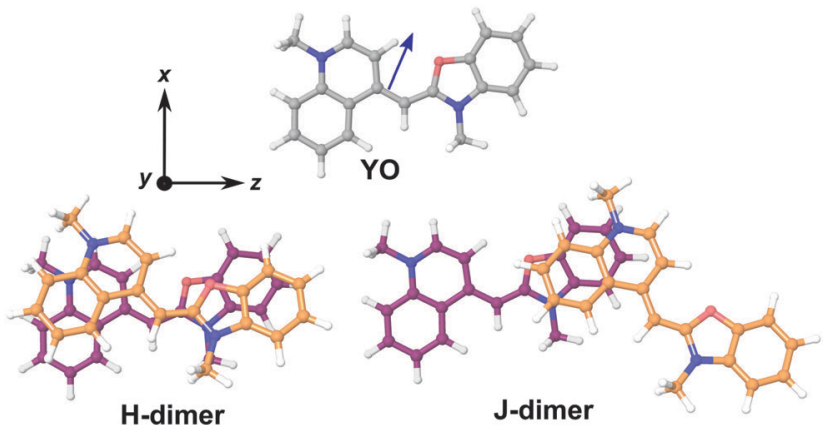

Fig. 7 Optimized structures of $\mathrm{YO}$ and the $\mathrm{H}$ - and $\mathrm{J}$-dimers. The $\mathrm{z}$-axis coincides with the $\mathrm{S}_{1} \leftarrow \mathrm{S}_{0}$ transition dipole moment of the YO monomer. For the dimers, the same orientation has been used for the YO unit in purple. The ground-state dipole moment (2.85 D) of $Y O$ is shown by the blue arrow.

slip angle (i.e. the angle between the stacking axis and the long molecular axis) is equal to $77^{\circ}$ and $38^{\circ}$, respectively. These mutual orientations found for both dimers result in substantial excitonic coupling, as shown by the TD-DFT calculations described below. Moreover, both dimers are far from centrosymmetric and thus their SSHG spectrum should be close to their linear absorption spectrum.

The assignment of these complexes to $\mathrm{H}$ - and J-dimers is based, besides geometrical considerations, on the results of TD-DFT calculations, which predict blue- and red-shifted electronic transitions relative to that of the monomer, respectively, (Fig. 8). Whereas the first vertical electronic transition of YO is calculated at $3.00 \mathrm{eV}$ with an oscillator strength of $f=1.06$, an intense absorption with $f=1.86$ is predicted at $3.08 \mathrm{eV}$ for the $\mathrm{H}$-dimer. On the other hand, the transition to the lowest excitonic state of the $\mathrm{H}$-dimer is calculated at $2.90 \mathrm{eV}$ with negligible intensity $(f=0.05)$. In the case of the J-dimer, an intense transition to the lower excitonic state with $f=1.84$ is predicted at $2.97 \mathrm{eV}$, whereas the transition to the upper excitonic state is at $3.02 \mathrm{eV}$ and is much weaker $(f=0.27)$. The non-vanishing oscillator strength of the transition to the upper excitonic state is due to an imperfect alignment of the two dyes in the J-dimer. The nature and structure of aggregates have been shown to critically depend on factors such as electrostatic interactions, size and geometry. ${ }^{70}$ For example, highly polar merocyanine dyes form $\pi$-stacks with a $3.5 \AA$ distance between the aromatic planes, a slip angle close to $60^{\circ}$ and an antiparallel arrangement, as revealed by crystallographic and 2D NMR measurements. $^{70}$ In our optimized H-dimer model, the distance between the aromatic planes is $4.9 \AA$, larger than in the merocyanine aggregate, most probably due to the electrostatic repulsions between the two positively charged YO units. The larger predicted slip angle of $77^{\circ}$, i.e. close to the value of $90^{\circ}$ for a perfect face-to-face geometry, can be explained by the less sterically hindered YO model. The departure from $90^{\circ} \mathrm{mini}-$ mizes the electrostatic repulsion associated with the permanent electric dipole of YO.

The $\overleftrightarrow{\beta}$ tensor elements of YO and the $\mathrm{H}$ - and J-dimers were calculated using fixed coordinates as presented in Fig. 7. 


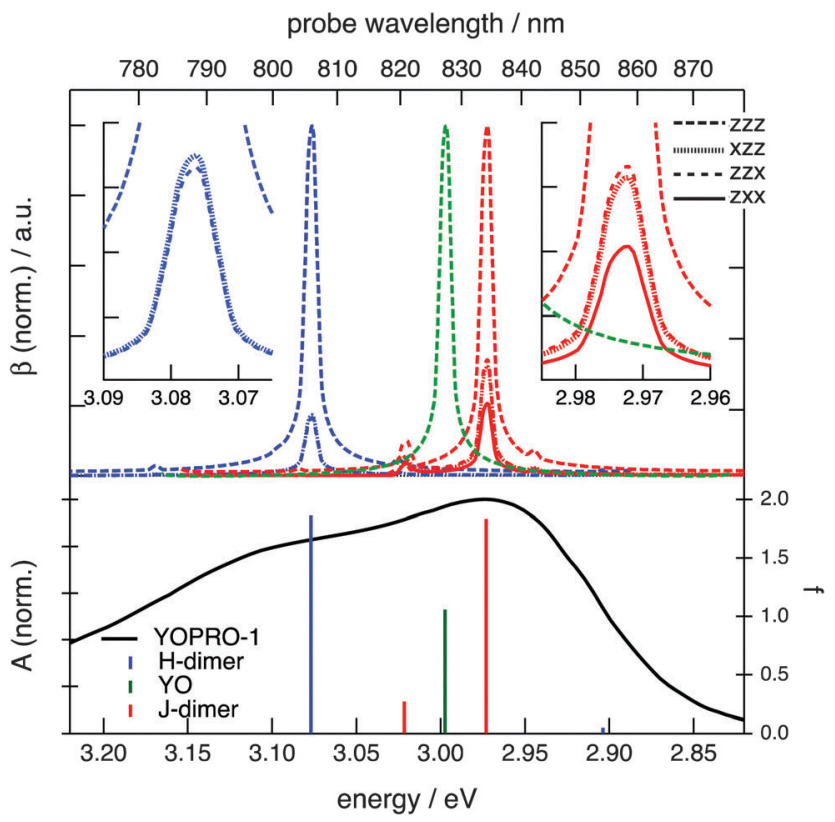

Fig. 8 (bottom) Calculated electronic transitions of $\mathrm{YO}, \mathrm{H}$ - and J-dimers in water. The solid line is the absorption spectrum of a $20 \mu \mathrm{M}$ solution of YOPRO-1 in water shifted to higher energy by $0.40 \mathrm{eV}$. (top) Spectral dependence of the main elements of the hyperpolarizability tensors of the monomer and dimers.

Fig. 8 shows the spectral dependence of the modulus of all major non-zero elements, $\left|\beta_{i j k}\right|$, normalized to the maximum of the most intense element of each tensor:

$$
\left|\beta_{i j k}\right|=\sqrt{\operatorname{Re}^{2}\left(\beta_{i j k}\right)+\operatorname{Im}^{2}\left(\beta_{i j k}\right)}
$$

The real and imaginary parts of these elements are plotted in Fig. S9 (ESI $\dagger$ ). The $\overleftrightarrow{\beta}$ tensor of YO is dominated by a single element, $\beta_{z z z}$, confirming the assumption made above in the analysis of the polarization-resolved experiments. This element is aligned along the $S_{1} \leftarrow S_{0}$ transition dipole moment and exhibits an intense two-photon resonance with this transition. The $\beta_{z z z}$ element is also the major one for the dimers, although other elements, namely $\beta_{x z z}$ and $\beta_{z z x}$, have a non-negligible magnitude. In this case as well, these tensor elements show a two-photon resonance with the transition to the higher and lower excitonic states of the $\mathrm{H}$ - and J-dimers, respectively.

The ratio between the total intrinsic hyperpolarizability of the dimers and the monomer, $\beta_{\text {tot }}(\operatorname{dim}) / \beta_{\text {tot }}($ mon $)$ has been estimated using $\beta_{\text {tot }}$ defined as: ${ }^{50}$

$$
\beta_{\mathrm{tot}}=\sqrt{\beta_{x}^{2}+\beta_{y}^{2}+\beta_{z}^{2}}
$$

where

$$
\beta_{i}=\beta_{i i i}+\frac{1}{3} \sum_{j \neq i}\left(\beta_{i j j}+\beta_{j i j}+\beta_{j j i}\right)
$$

This ratio, calculated at the resonance frequency, amounts to 1.39 and 1.72 for the $\mathrm{H}$ - and J-dimers, respectively. Similar ratios are calculated at a frequency far from resonance, namely 1.49 and 1.71, respectively, at $3.13 \mathrm{eV}$.

These calculations confirm that both $\mathrm{H}$ - and J-type YO dimers have a second-order optical response, which is comparable in magnitude with that of a single YO molecule. Therefore, the SSHG band observed at around $450 \mathrm{~nm}$ with the YOSAC dyes can unambiguously be ascribed to a H-dimer or a higher H-aggregate.

The absence of a H-dimer band in the SSHG spectrum of YOYO-1 cannot be ascribed to a vanishingly small nonlinear response, as this would require a mutual orientation of the chromophoric units resulting in a $\mathrm{H}$-dimer with a centre of inversion. Such geometry cannot be realized with YOYO-1 because of the constraints imposed by the linker. Therefore, the lack of aggregate SSHG band indicates that YOYO-1 does not exist in the $\mathrm{H}$-dimer form at the interface. This is due to the distribution of electric charges in the molecule that favors a rather flat orientation of the chromophore on the interface and thus prevents the formation of $\mathrm{H}$-dimers. The latter would require a smaller tilt angle, similar to that found for YOSAC-1.

\section{Time-resolved SSHG}

Further insight into the properties of the YO dyes adsorbed at the interfaces was obtained from TR-SSHG measurements. Fig. 9A shows the time-profiles of the SSHG signal intensity, $S(t)$, recorded at $485 \mathrm{~nm}$ upon $500 \mathrm{~nm}$ excitation using s/p and $\mathrm{m} / \mathrm{s}$ polarizations. As the SSHG signal at this wavelength is due to a two-photon resonance with the $S_{1} \leftarrow S_{0}$ transition, the decrease of the SSHG intensity upon photoexcitation reflects the depletion of the ground-state population. The recovery of the signal can be due to two processes: (i) the decay of the excited-state population and (ii) the out-of-plane motion that suppresses the orientational anisotropy due to the excitation with circularly polarized light. Such out-of-plane motion has been shown to take place on hundreds of picoseconds timescale. ${ }^{71}$

In the present case, the signal recovery occurs on different timescales and can be reproduced with the sum of two exponential functions (solid lines), with time constants of $\sim 15-20 \mathrm{ps}$ and $>1$ ns. Although these time constants are similar for both polarization geometries, the relative amplitudes differ, with that associated with the fast component decreasing from 0.74 to 0.60 when going from $\mathrm{s} / \mathrm{p}$ to $\mathrm{m} / \mathrm{s}$ geometry. According to eqn (2), the SSHG intensity at those polarizations should reflect $\chi_{Z X X}^{(2)}$ and $\chi_{X X Z}^{(2)}$, respectively. As the quantum chemical calculations evidenced that the hyperpolarizability tensor of $\mathrm{YO}$ is dominated by a single element, both $\chi_{Z X X}^{(2)}$ and $\chi_{X X Z}^{(2)}$ should be identical, even in the presence of orientational anisotropy, ${ }^{71}$ and given by: ${ }^{68}$

$$
\chi_{Z X X}^{(2)}=\chi_{X X Z}^{(2)}=\frac{1}{2} N\left\langle\sin ^{2} \theta \cos \theta\right\rangle \beta_{z z z}
$$

As a consequence, if the SSHG signal at this wavelength was only due to a single species or two different species having a dominating $\beta_{z z z}$ element, both time profiles should, in principle, be identical. Therefore, the difference observed here should arise from the presence of another form of YOYO-1 
apart from that with the non-interacting YO units, but which is not a H-dimer, as evidenced by the absence of the SSHG band. This form, which will be called $\mathrm{X}$ as its exact nature is unknown, should have a geometry allowing some interaction between the YO units and resulting in a $\overleftrightarrow{\beta}$ tensor that differs from that with non-interacting units. However, excitonic coupling should not be very significant, as testified by the negligible polarization dependence of the SSHG spectrum of YOYO-1 (Fig. S5, ESI $\dagger$ ) and by the TR-SSHG profile measured at $460 \mathrm{~nm}$, which is similar to that at $485 \mathrm{~nm}$ but with a slightly larger amplitude of the fast component, i.e. 0.78 vs. 0.74 (Fig. S10, ESI $\dagger$ ). For example, the YOYO-1 structure with close to perpendicular mutual orientation of the chromophores, as that observed when the dye is intercalated into DNA, would result in a small excitonic coupling. The $\overleftrightarrow{\beta}$ tensor associated with this structure should differ from that of the monomer but the resonances should be essentially the same.

The fast recovery component is ascribed to the YOYO-1 population with non-interacting units, whereas the slow component is attributed to the $\mathrm{X}$ form. This assignment is based on previous investigations of YOYO-1 in aqueous solutions that reported excited-state lifetimes of $4 \mathrm{ps}$ and $1.1 \mathrm{~ns}$ for the nonaggregated and $\mathrm{H}$-dimer forms, respectively. ${ }^{8}$ The longer excitedstate lifetime of the dimer originates from the hindrance that arises upon $\pi$ stacking of the large amplitude motion responsible for the non-radiative decay. A similar explanation can be proposed here to account for the slow recovery dynamics of the $\mathrm{X}$ form.

The 4-5 times larger time constant found here for the excited-state decay of the non-interacting form is probably due to the intrinsic properties of the interface. As observed with many other flexible chromophores, ${ }^{72}$ the excited-state dynamics of YOYO-1 and other YO derivatives in solution is dominated by a non-radiative decay process involving intramolecular modes with large amplitude motion. The time constant associated with this process, $\tau_{\mathrm{nr}}$, varies with the viscosity of the environment, $\eta$, as $\tau_{\mathrm{nr}} \propto \eta^{\alpha}$, where $\alpha$ depends on the nature of the intramolecular modes involved in the non-radiative decay, and ranges usually between $\sim 0.3$ and $1 .^{73}$ According to the data reported for YOPRO-1 and YOSAC- 1 in four different solvents, ${ }^{7} \alpha$ can be estimated to be around 1. Therefore, the friction experienced by YOYO- 1 at the dodecane/water interface can be estimated to be around 4-5 times as large as in bulk water. This observation agrees well with the lifetime increase reported for malachite green when going from bulk water $(\tau=0.6 \mathrm{ps})$ to the dodecane/ water interface $(\tau=2.5 \mathrm{ps}) .{ }^{74}$ Considering that dodecane is more viscous than water by a factor of 1.5 only, the larger excited-state lifetimes at the interface cannot be accounted by the protrusion of the adsorbed dyes in the apolar phase, ${ }^{26}$ and should reflect an intrinsic property of the interface. Higher interfacial friction has also been observed at the air/water interface, ${ }^{63,75,76}$ and has been ascribed to the stiffer nature of interfacial water, owing to a stronger hydrogen-bond network.

Fig. 9B reveals that the TR-SSHG profile at $485 \mathrm{~nm}$ measured with YOPRO-1 at the dodecane/PBS interface is almost entirely

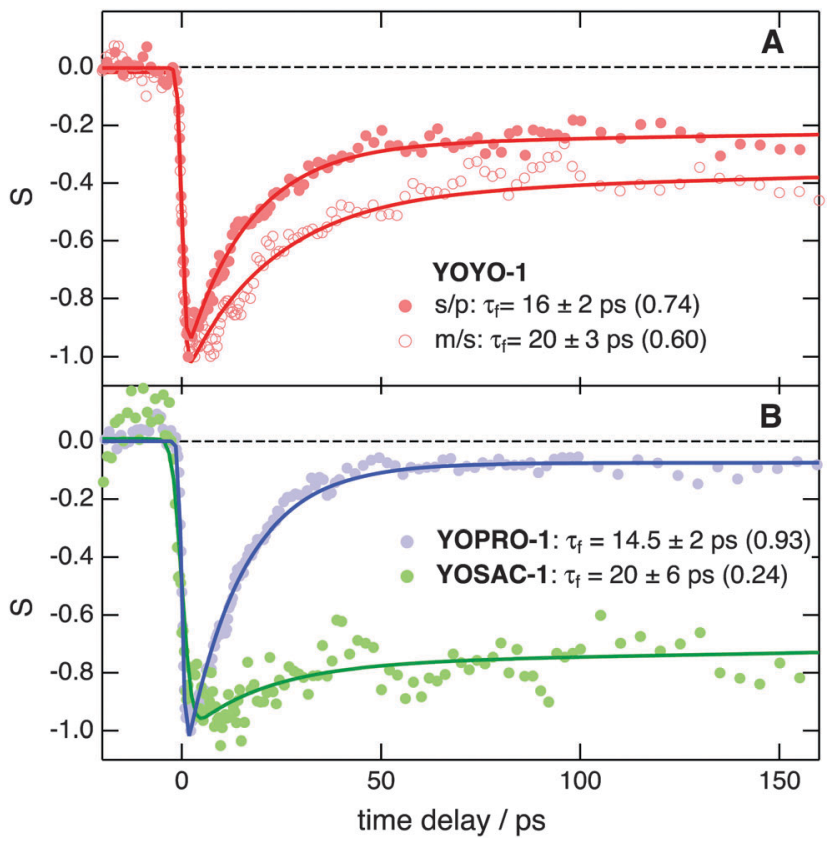

Fig. 9 (A) TR-SSHG profiles, S(t), measured with (A) YOYO-1 (10 $\mu \mathrm{M})$ at $485 \mathrm{~nm}$ at the dodecane/water interface using two polarization geometries and (B) a comparison of the TR-SSHG profiles measured at $485 \mathrm{~nm}$ using the $s / p$ polarization with YOSAC-1 $(10 \mu \mathrm{M})$ at the dodecane/water interface and with YOPRO-1 $(10 \mu \mathrm{M})$ at the dodecane/PBS interface. The pump pulses were at $500 \mathrm{~nm}$. The solid lines are the best bi-exponential fits $\left(\tau_{\mathrm{f}}\right.$ is the time constant of the fast component and its relative amplitude is given in parentheses; the time constant of the slow component is $>1 \mathrm{~ns}$ ).

dominated by the fast component (relative amplitude of 0.93) with a time constant of $14.5 \pm 2 \mathrm{ps}$, similar to that associated with the fast recovery component of YOYO-1. Upon doubling the bulk concentration of YOPRO-1 from 10 to $20 \mu \mathrm{M}$, the amplitude of this slow component increases to 0.1 , but the short time constant remains essentially unchanged (Fig. S11, $\mathrm{ESI} \dagger$ ). At $475 \mathrm{~nm}$, the slow component is no longer present and the time profile recorded at the same concentration can be reproduced with a single exponential function with a $15.5 \pm 2 \mathrm{ps}$ time constant (Fig. S12, ESI $\dagger$ ). These results are in good agreement with the stationary SSHG measurements showing that YOPRO-1 does not form H-aggregates at the interface. However, the presence of a slow recovery component whose amplitude increases with bulk concentration points to the formation of another type of aggregate having properties similar to those found for the $\mathrm{X}$ form of YOYO-1.

Finally, Fig. 9B indicates that, contrary to YOYO-1 and YOPRO-1, the ground-state recovery dynamics of YOSAC-1 is dominated by the slow component, whose relative amplitude amounts to 0.76. This result is in accordance with the SSHG spectrum (Fig. 4C) that points to a large population of $\mathrm{H}$-aggregates at the interface.

In summary, these TR-SSHG measurements confirm the high propensity of YOSAC-1 to form H-aggregates at the interface. They also reveal that, although YOYO-1 and YOPRO-1 do not exist as a H-dimer/aggregate at the interface, they exist in 
two different forms: a predominant one where the two chromophores are far apart and do not interact and another where the mutual orientation of the chromophoric units is such that it minimizes the excitonic interaction and inhibits the nonradiative decay of the excited state by large-amplitude motion.

\section{Conclusions}

We have presented here the first SSHG study, to the best of our knowledge, of the well-known YO fluorescent DNA probes at liquid/liquid interfaces. As anticipated from their remarkable performance as DNA intercalators, these dyes, especially the singly charged ones, exhibit a high affinity for the dodecane/ water interface, and generate a large SSHG signal in the 440-500 nm region owing to a two-photon electronic resonance. The ensemble of experimental data reveals an interfacial behavior that depends strongly on the structure of the dye, especially its electric charge. The only homodimeric dye investigated here, YOYO-1, exhibits a behavior upon adsorption that is similar to that observed in bulk aqueous solutions upon addition of DNA. Indeed, the YOYO-1 H-dimer, which is predominant in water, is disrupted upon adsorption at the interface as well as upon intercalation into DNA. Because of the absence of a strong lipophilic group, the YO units adopt a rather flat orientation at the interface that does not favor $\pi$-stacking and thus inhibits the formation of H-dimers. However, some interaction between the $\mathrm{YO}$ units that hinders large-amplitude motion around the monomethine bond and hence lengthens the excitedstate lifetime of the dye, can still take place at the interface. As a consequence, these results predict an increase of the fluorescence quantum yield of YOYO-1 upon adsorption at the interface. This aspect, which still needs to be verified, would confer YOYO-1 interesting properties as a fluorescent interfacial probe.

On the other hand, the interfacial behavior of the monomeric dyes, i.e. YOPRO-1, YOSAC-1 and YOSAC-3, does not differ much from that observed in aqueous solution. In both cases, YOPRO-1 exhibits little tendency for $\mathrm{H}$-aggregation, which can be explained by its doubly charged nature that favors neither adsorption nor aggregation. Some intermolecular interactions, which seem to be of a similar nature than the intramolecular interactions measured with YOYO-1, are operative with this dye at the interface. Being singly charged, the YOSAC dyes exhibit a higher affinity for the interface, where they adopt a more perpendicular orientation than the doubly charged dyes. Both this orientation and the smaller charge promote $\pi$ stacking and the formation of $\mathrm{H}$-aggregates. As the latter are not emissive, the YOSAC dyes should not be selected as fluorescent interfacial probes. However, given their large SSHG signal and high interfacial activity, they could be used as probes in SHG microscopy.

Very preliminary SSHG measurements in the presence of DNA in the aqueous phase indicate that these YO dyes can also be used to study the interfacial properties of nucleic acids. This approach might prove to be useful for investigating biomolecules in heterogeneous environments.

\section{Acknowledgements}

The authors wish to thank Prof. T. Deligeorgiev (Faculty of Chemistry, University of Sofia) for supplying the fluorescent dyes. This work was supported by the Swiss National Science Foundation through project no. 200020-147098 and the University of Geneva.

\section{References}

1 A. N. Glazer and H. S. Rye, Nature, 1992, 359, 859-861.

2 H. S. Rye, S. Yue, M. A. Quesada, R. P. Haugland, R. A. Mathies and A. N. Glazer, Methods Enzymol., 1993, 217, 414-431.

3 C. Carlsson, A. Larsson, M. Jonsson, B. Albinsson and B. Norden, J. Phys. Chem., 1994, 98, 10313-10321.

4 T. L. Netzel, K. Nafisi, M. Zhao, J. R. Lenhard and I. Johnson, J. Phys. Chem., 1995, 99, 17936-17947.

5 L. Mikelsons, C. Carra, M. Shaw, C. Schweitzer and J. C. Scaiano, Photochem. Photobiol. Sci., 2005, 4, 798-802.

6 A. Fürstenberg, T. G. Deligeorgiev, N. I. Gadjev, A. A. Vasilev and E. Vauthey, Chem. - Eur. J., 2007, 13, 8600-8609.

7 A. Fürstenberg and E. Vauthey, J. Phys. Chem. B, 2007, 111, 12610-12620.

8 A. Fürstenberg, M. D. Julliard, T. G. Deligeorgiev, N. I. Gadjev, A. A. Vassilev and E. Vauthey, J. Am. Chem. Soc., 2006, 128, 7661-7669.

9 M. Kasha, H. R. Rawls and M. A. El-Bayoumi, Pure Appl. Chem., 1965, 11, 371-392.

10 V. Sundstroem and T. Gillbro, J. Chem. Phys., 1985, 83, 2733-2743.

11 I. Renge and U. P. Wild, J. Phys. Chem. A, 1997, 101, 7977-7988.

12 R. F. Khairutdinov and N. Serpone, J. Phys. Chem. B, 1997, 101, 2602-2610.

13 J. L. Seifert, R. E. Connor, S. A. Kushon, M. Wang and B. A. Armitage, J. Am. Chem. Soc., 1999, 121, 2987-2995.

14 M. Wang, G. L. Silva and B. A. Armitage, J. Am. Chem. Soc., 2000, 122, 9977-9986.

15 T. D. Slavnova, A. K. Chibisov and H. Gorner, J. Phys. Chem. A, 2005, 109, 4758-4765.

16 S. Gadde, E. K. Batchelor, J. P. Weiss, Y. Ling and A. E. Kaifer, J. Am. Chem. Soc., 2008, 130, 17114-17119.

17 S. M. Mooi, S. N. Keller and B. Heyne, Langmuir, 2014, 30, 9654-9662.

18 N. A. Toropov, P. S. Parfenov and T. A. Vartanyan, J. Phys. Chem. C, 2014, 118, 18010-18014.

19 J. S. Schildkraut, T. L. Penner, C. S. Willand and A. Ulman, Opt. Lett., 1988, 13, 134-136.

20 N. Kato, K. Saito and Y. Uesu, Thin Solid Films, 1999, 338, 5-8.

21 Y. Niidome, H. Ayukawa and S. Yamada, J. Photochem. Photobiol., A, 2000, 132, 75-80.

22 G. Janssens, F. Touhari, J. W. Gerritsen, H. van Kempen, P. Callant, G. Deroover and D. Vandenbroucke, Chem. Phys. Lett., 2001, 344, 1-6. 
23 R. Adachi, T. Inoue and T. Ogawa, Anal. Sci., 2001, 17, 449-451.

24 A. Yamaguchi, M. Nakano, K. Nochi, T. Yamashita, K. Morita and N. Teramae, Anal. Bioanal. Chem., 2006, 386, 627-632.

25 G. Martin-Gassin, E. Benichou, G. Bachelier, I. RussierAntoine, C. Jonin and P. F. Brevet, J. Phys. Chem. C, 2008, 112, 12958.

26 G. Martin-Gassin, D. Villamaina and E. Vauthey, J. Am. Chem. Soc., 2011, 133, 2358-2361.

27 D. A. Steinhurst, A. P. Baronavski and J. C. Owrutsky, J. Phys. Chem. B, 2002, 106, 3160-3165.

28 H. Nagatani, A. Piron, P.-F. Brevet, D. J. Fermin and H. H. Girault, Langmuir, 2002, 18, 6647-6652.

29 K. Fujiwara, S. Wada, H. Monjushiro and H. Watarai, Langmuir, 2006, 22, 2482-2486.

30 M. Fedoseeva, P. Fita, A. Punzi and E. Vauthey, J. Phys. Chem. C, 2010, 114, 13774-13781.

31 M. Fedoseeva, R. Letrun and E. Vauthey, J. Phys. Chem. B, 2014, 118, 5184-5193.

32 S. Yamaguchi and T. Tahara, J. Chem. Phys., 2006, 125, 194711.

33 J. Massin, A. Charaf-Eddin, F. Appaix, Y. Bretonnière, D. Jacquemin, B. van der Sanden, C. Monnereau and C. Andraud, Chem. Sci., 2013, 4, 2833.

34 D. P. Mahoney, E. A. Owens, C. Fan, J. C. Hsiang, M. M. Henary and R. M. Dickson, J. Phys. Chem. B, 2015, 119, 4637-4643.

35 H. Chen, W. Lin, H. Cui and W. Jiang, Chem. - Eur. J., 2015, 21, 733-745.

36 T. Deligeorgiev, N. Gadjev, A. Vasilev, K. Drexhage and S. Yarmoluk, Dyes Pigm., 2006, 70, 185-191.

37 N. I. Gadjev, T. G. Deligeorgiev, I. Timcheva and V. Maximova, Dyes Pigm., 2003, 57, 161-164.

38 M. Fedoseeva, P. Fita and E. Vauthey, Langmuir, 2013, 29, 14865-14872.

39 S. Richert, S. Mosquera Vazquez, M. Grzybowski, D. T. Gryko, A. Kyrychenko and E. Vauthey, J. Phys. Chem. B, 2014, 118, 9952-9963.

40 M. J. Frisch, G. W. Trucks, H. B. Schlegel, G. E. Scuseria, M. A. Robb, J. R. Cheeseman, G. Scalmani, V. Barone, B. Mennucci, G. A. Petersson, H. Nakatsuji, M. Caricato, X. Li, H. P. Hratchian, A. F. Izmaylov, J. Bloino, G. Zheng, J. L. Sonnenberg, M. Hada, M. Ehara, K. Toyota, R. Fukuda, J. Hasegawa, M. Ishida, T. Nakajima, Y. Honda, O. Kitao, H. Nakai, T. Vreven, J. A. Montgomery Jr., J. E. Peralta, F. Ogliaro, M. Bearpark, J. J. Heyd, E. Brothers, K. N. Kudin, V. N. Staroverov, R. Kobayashi, J. Normand, K. Raghavachari, A. Rendell, J. C. Burant, S. S. Iyengar, J. Tomasi, M. Cossi, N. Rega, J. M. Millam, M. Klene, J. E. Knox, J. B. Cross, V. Bakken, C. Adamo, J. Jaramillo, R. Gomperts, R. E. Stratmann, O. Yazyev, A. J. Austin, R. Cammi, C. Pomelli, J. W. Ochterski, R. L. Martin, K. Morokuma, V. G. Zakrzewski, G. A. Voth, P. Salvador, J. J. Dannenberg, S. Dapprich, A. D. Daniels, Ö. Farkas, J. B. Foresman, J. V. Ortiz, J. Cioslowski and D. J. Fox, Gaussian 09 (revision C1), Wallingford, CT, 2010.
41 A. D. Becke, Phys. Rev. A: At., Mol., Opt. Phys., 1988, 38, 3098-3100.

42 C. Lee, W. Yang and R. G. Parr, Phys. Rev. B: Condens. Matter Mater. Phys., 1988, 37, 785-789.

43 T. Yanai, D. P. Tew and N. C. Handy, Chem. Phys. Lett., 2004, 393, 51-57.

44 D. Jacquemin, E. A. Perpete, G. Scalmani, M. J. Frisch, R. Kobayashi and C. Adamo, J. Chem. Phys., 2007, 126, 144105.

45 P. A. Limacher, K. V. Mikkelsen and H. P. Luthi, J. Chem. Phys., 2009, 130, 194114.

46 M. Medved', Š. Budzák and T. Pluta, Chem. Phys. Lett., 2011, 515, 78-84.

47 A. Alparone, Adv. Phys. Chem., 2013, 2013, 1-8.

48 G. Maroulis and A. J. Thakkar, J. Chem. Phys., 1989, 90, 366 .

49 E. F. Archibong and A. J. Thakkar, Chem. Phys. Lett., 1993, 201, 485-492.

50 D. R. Kanis, M. A. Ratner and T. J. Marks, Chem. Rev., 1994, 94, 195-242.

51 A. V. Marenich, C. J. Cramer and D. G. Truhlar, J. Phys. Chem. B, 2009, 113, 6378-6396.

52 R. Bauernschmitt and R. Ahlrichs, Chem. Phys. Lett., 1996, 256, 454-464.

53 R. E. Stratmann, G. E. Scuseria and M. J. Frisch, J. Chem. Phys., 1998, 109, 8218-8224.

54 G. Scalmani, M. J. Frisch, B. Mennucci, J. Tomasi, R. Cammi and V. Barone, J. Chem. Phys., 2006, 124, 94107.

55 H. Sekino and R. J. Bartlett, J. Chem. Phys., 1986, 85, 976-989.

56 J. E. Rice and N. C. Handy, J. Chem. Phys., 1991, 94, 4959-4971.

57 J. E. Rice and N. C. Handy, Int. J. Quantum Chem., 1992, 43, 91-118.

58 S. Yamaguchi and T. Tahara, J. Phys. Chem. B, 2004, 108, 19079-19082.

59 N. S. Makarov, M. Drobizhev and A. Rebane, Opt. Express, 2008, 16, 4029-4047.

60 C. Xu and W. W. Webb, J. Opt. Soc. Am. B, 1996, 13, 481-491.

61 L. Goodman and R. P. Rava, Acc. Chem. Res., 1984, 17, 250-257.

62 I. Benjamin, Chem. Rev., 2006, 106, 1212-1233.

63 A. Punzi, G. Martin-Gassin, J. Grilj and E. Vauthey, J. Phys. Chem. C, 2009, 113, 11822-11829.

64 K. B. Eisenthal, Chem. Rev., 1996, 96, 1343-1360.

65 A. A. Tamburello-Luca, P. Hebert, P. F. Brevet and H. H. Girault, J. Chem. Soc., Faraday Trans., 1996, 92, 3079-3085.

66 G. J. Simpson, S. G. Westerbuhr and K. L. Rowlen, Anal. Chem., 2000, 72, 887-898.

67 B. Doughty, Y. Rao, S. W. Kazer, S. J. Kwok, N. J. Turro and K. B. Eisenthal, Proc. Natl. Acad. Sci. U. S. A., 2013, 110, 5756-5758.

68 P.-F. Brevet, Surface Second Harmonic Generation, Presses polytechniques et universitaires romandes, Lausanne, 1997. 
69 J. Rinuy, A. Piron, P. F. Brevet, M. Blanchard-Desce and H. H. Girault, Chem. - Eur. J., 2000, 6, 3434-3441.

70 Z. Chen, A. Lohr, C. R. Saha-Moller and F. Wurthner, Chem. Soc. Rev., 2009, 38, 564-584.

71 D. Zimdars, J. I. Dadap, K. B. Eisenthal and T. F. Heinz, J. Phys. Chem. B, 1999, 103, 3425-3433.

72 A. Rosspeintner, B. Lang and E. Vauthey, Annu. Rev. Phys. Chem., 2013, 64, 247-271.
73 S. P. Velsko and G. R. Fleming, Chem. Phys., 1982, 65, 59-70.

74 P. Fita, A. Punzi and E. Vauthey, J. Phys. Chem. C, 2009, 113, 20705-20712.

75 X. Shi, E. Borguet, A. N. Tarnovsky and K. B. Eisenthal, Chem. Phys., 1996, 205, 167-178.

76 P. Sen, S. Yamaguchi and T. Tahara, Faraday Discuss., 2010, 145, 411-428. 